\title{
Ocular pulse amplitude is reduced in patients with advanced retinitis pigmentosa
}

\author{
Karl-Georg Schmidt, Lutz E Pillunat, Konrad Kohler, Josef Flammer
}

\begin{abstract}
Backgroundlaims-The choroid, a low resistance vascular structure carrying $85 \%$ of the ocular blood flow, provides nourishment to and removal of potential toxic waste products from the adjacent non-vascularised outer layers of the retina, macula, and optic disc regions. Choroidal perfusion may be reduced in retinitis pigmentosa (RP) and might contribute to retinal pigment epithelium (RPE) degeneration. The aim of this study was to determine whether choroidal perfusion is reduced in RP and whether this is correlated with the stage of disease.

Methods-Ocular pulse amplitude (OPA) evaluated with the ocular blood flow (OBF) system, applanation intraocular pressure (IOP), visual fields, blood pressure (BP), and heart rate (HR) were measured in $75 \mathrm{RP}$ patients having stage RP-I (stage I: visual field size: 7.85-14.67 $\mathbf{c m}^{2} ; \mathbf{n}=22$ ), stage RP-II (stage II: visual field size: $2.83-7.84 \mathrm{~cm}^{2} ; n=29$ ), or stage RP-III (stage III: visual field size: 0.52$\left.2.82 \mathrm{~cm}^{2} ; \mathrm{n}=24\right)$ were compared with matched healthy controls and each other. Results-Neither IOP nor systemic perfusion parameters were significantly ( $p$ $>0.1$ ) altered, but OPA ( $\mathrm{mm} \mathrm{Hg}$ ) in RP patients beginning with stage RP-II (1.6 (0.1), 27.3\%, p<0.0001), and RP-III (1.2 $(0.1), 45.5 \%, p<0.0001)$ was significantly reduced when compared with matched subgroups from a pool of healthy controls (2.2 (0.1), $\mathrm{n}=94)$.

Conclusions-OPA can be used neither for early clinical detection of RP nor to follow the natural course of the disease. However, our data show that in advanced stages of RP not only the retina but also the choroidal circulation is affected.

(Br f Ophthalmol 2001;85:678-682)
\end{abstract}

Retinitis pigmentosa (RP) comprises a group of progressive degenerative retinal diseases caused by genetic defects localised on different chromosomes affecting approximately 1.5 million people worldwide. In the majority of cases, $\mathrm{RP}$ leads to blindness at around the age of 60 years. ${ }^{1}$

Whereas retinal perfusion is reduced in $\mathrm{RP}{ }^{2}$ possibly related to a difference in the electrophysiological properties of retinal capillary pericytes, ${ }^{3}$ a review of the literature shows contradictory results regarding the role of the choroidal vasculature in this genetic aberration. Histological examination showed a normal choroid in two donor eyes, ${ }^{4}$ while Henkind and Gartner observed absence of the choriocapillaris. ${ }^{5}$ Ulrich et al using perfusion pressure videoangiography showed atrophy of the choroid capillary network. ${ }^{6}$ Langham and Kramer noted an overall $48 \%$ reduction of the mean ocular pulse amplitude in $13 \mathrm{RP}$ patients with varying degrees of disease when compared with healthy volunteers. ${ }^{7}$

The choroid is a low resistance vascular structure which maintains a constant thermal environment for the retina, ${ }^{89}$ as well as supplying $80 \%$ of the total retinal oxygen, ${ }^{8}{ }^{9}$ all the retinal glucose, ${ }^{10}$ and $100 \%$ of the nutrients to the macula and optic disc region. ${ }^{11}$ It is also responsible for the removal of potential toxic waste products from the retina. Nutrition and clearance of the retina by diffusion necessitate an extremely high blood flow in the choroid $\left(800-1200 \mathrm{ml} / 100 \mathrm{~g} / \mathrm{min},{ }^{12}\right.$ which reflects $85 \%$ of the total ocular blood flow). ${ }^{11}$ Reduced blood flow is correlated with hypoxia in an extreme situation resulting in, for example, myocardial or cerebral tissue infarctions. Similar cell death mechanisms might also exist in ocular pathology, such as glaucoma and RP. Results from clinical studies show reduced choroidal perfusion in normal tension glaucoma (NTG) patients and increased choroidal perfusion in ocular hypertensive volunteers pointing at a protective mechanism or adaptive change to prevent hypoxia of neural tissue because reduced blood flow might result in functional (visual field defects) and morphological (nerve fibre layer loss) damage. ${ }^{13} 14$

Vasoactive drugs increase choroidal perfusion in $\mathrm{NTG}^{15}{ }^{16}$ and high tension glaucoma (HTG) patients, ${ }^{17}{ }^{18}$ and improve visual fields. ${ }^{19}$ Improved ocular haemodynamics and a beneficial effect on the remaining visual field area on breathing carbon dioxide, a strong vasodilator, has already been demonstrated in RP patients. $^{20}$

This study was designed to evaluate ocular pulse amplitude (OPA), a measure for choroidal blood flow in RP, and to correlate these data with visual field size.

Materials and methods

Included in the investigation were $75 \mathrm{RP}$ patients with no known systemic or ocular disease other than the category for which they were selected. Patients and healthy controls were on no ocular or systemic therapy.

The RP patients chosen had a history of pretreatment IOPs $<21 \mathrm{~mm} \mathrm{Hg}$, typical concentric visual field loss as determined by standard Goldmann perimetry (III, 4e) and electroretinographic findings (diminished or extinguished b-wave amplitude), and showed typical fundus appearance. 
Visual field size of all patients and controls was determined by the same well trained and highly motivated and experienced perimetrist along different radii in a random and rhythmic pattern using standard Goldmann perimetry (III, $4 \mathrm{e}$, stimulus movement at $2^{\circ}$ per second) scanning the visual fields into a $166 \mathrm{MHz}$ computer (Fujitsu America Inc, San Jose, CA, USA) and calculating the area within the III, 4 margin using the Autocad R 12 program (Autodesk Systems Inc, San Rafael, CA, USA); visual field size reduction was expressed in percentages when compared with our standard of a healthy visual field $\left(15.10(0.06) \mathrm{cm}^{2}\right.$, $0 \%$ visual field size reduction, $\mathrm{n}=94$, age 23.3 (2.2) years).

The RP patients were staged according to visual field size related to patient self assessment: RP-I (stage I: visual field size: 7.85 $14.67 \mathrm{~cm}^{2}$, respective visual field size reduction $2.9-48.0 \% ; \mathrm{n}=22$, male $=12$, female $=10$,

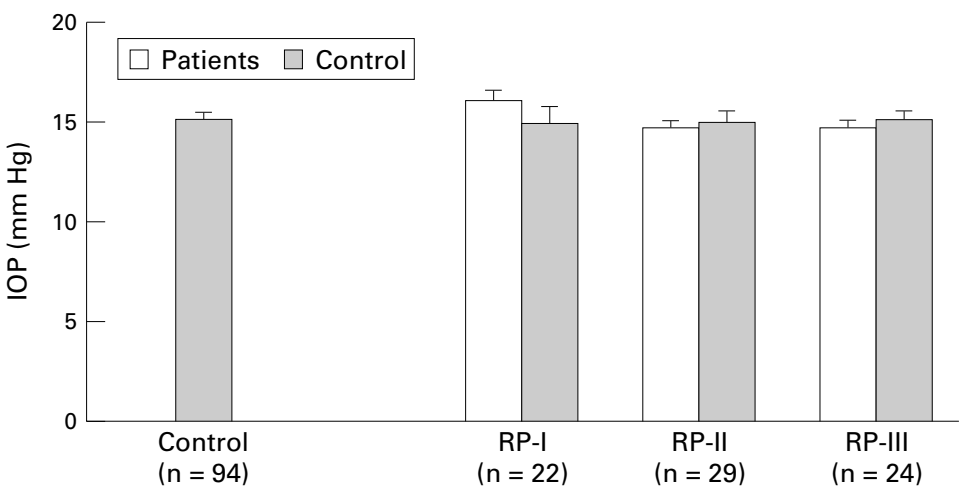

Figure 1 Intraocular pressures (IOP; mean (SEM)) of retinitis pigmentosa (RP) patients stages $I(n=22), I I(n=29)$, and III $(n=24)$, each compared with a matched control subgroup from a pool of healthy volunteers $(n=94)$.

Table 1 Comparison of perfusion parameters (mean (SEM)) of retinitis pigmentosa (RP) patients stages I $(n=22), I I(n=29)$, and III $(n=24)$, each compared with a matched control subgroup from a pool of healthy volunteers $(n=94)$

\begin{tabular}{llllll}
\hline & $\begin{array}{l}\mathrm{OPA} \\
(\mathrm{mm} \mathrm{Hg})\end{array}$ & $\begin{array}{l}\text { IOP } \\
(\mathrm{mm} \mathrm{Hg})\end{array}$ & $\begin{array}{l}\text { Blood pressure } \\
\text { (systolic, } m m \text { Hg) }\end{array}$ & $\begin{array}{l}\text { Blood pressure } \\
\text { (diastolic, } m m \text { Hg) }\end{array}$ & $\begin{array}{l}\text { Heart rate } \\
\text { (pulse, beats/min) }\end{array}$ \\
\hline Control & $2.2(0.1)$ & $15.0(0.3)$ & $124.8(1.6)$ & $77.1(1.3)$ & $76.8(1.6)$ \\
RP-I & $2.2(0.1)$ & $16.1(0.5)$ & $121.0(4.2)$ & $76.2(2.3)$ & $76.2(4.2)$ \\
Matched control & $2.2(0.2)$ & $14.9(0.8)$ & $123.4(5.9)$ & $76.8(1.9)$ & $78.0(5.0)$ \\
RP-II & $1.6(0.1)^{\star}$ & $14.7(0.4)$ & $123.3(2.7)$ & $77.2(1.5)$ & $80.0(1.9)$ \\
Matched control & $2.2(0.1)$ & $15.0(0.6)$ & $127.1(4.2)$ & $75.7(2.0)$ & $76.3(2.5)$ \\
RP-III & $1.2(0.1)^{\star}$ & $14.7(0.3)$ & $127.6(4.1)$ & $79.8(3.1)$ & $80.7(4.0)$ \\
Matched control & $2.3(0.1)$ & $15.1(0.4)$ & $124.5(3.7)$ & $76.4(2.6)$ & $76.7(3.2)$ \\
\hline
\end{tabular}

$\star$ Represents $\mathrm{p}<0.05$ using Student's unpaired two tailed $t$ test.

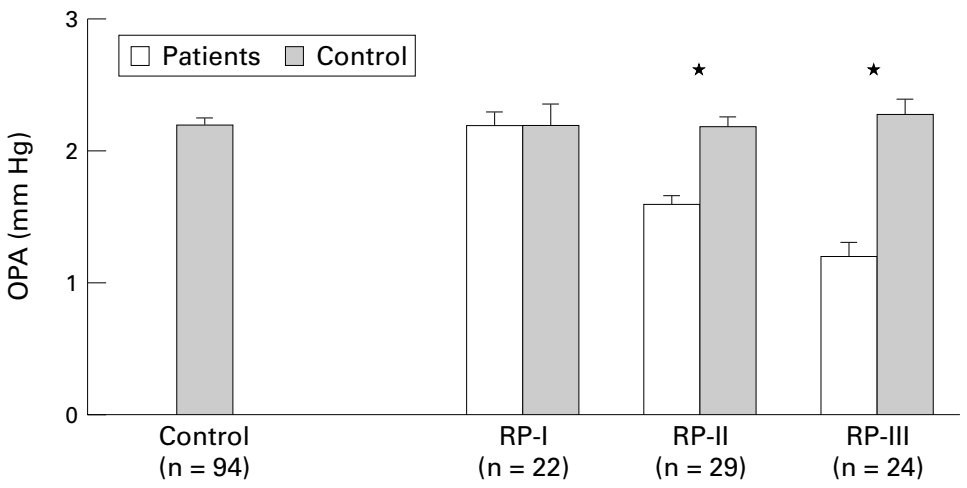

Figure 2 Ocular pulse amplitudes (OPA; mean (SEM)) of retinitis pigmentosa (RP) patients stages $I(n=22), I I(n=29)$, and III $(n=24)$, each compared with a matched control subgroup from a pool of healthy volunteers $(n=94)$. ${ }^{\star}$ Represents $p<0.05$ using Student's unpaired two tailed t test. age: 25.1 (2.2) years), RP-II (stage II: visual field size: $2.83-7.84 \mathrm{~cm}^{2}$, respective visual field size reduction: $48.1-81.2 \% ; \mathrm{n}=29$, male $=17$, female $=12$, age: 33.3 (2.8) years), and RP-III (stage III: visual field size: $0.52-2.82 \mathrm{~cm}^{2}$, respective visual field size reduction: 81.3$96.6 \% ; \mathrm{n}=24$, male $=14$, female $=10$, age: 47.7 (3.9) years).

Patient groups were compared with matched control subgroups selected from a pool of 94 healthy volunteers with no known ocular or systemic disease, who showed no visual field defects and/or disc alterations and had IOPs $<21 \mathrm{~mm} \mathrm{Hg}$ matched for age (SD 3 years), sex, IOP (SD $2 \mathrm{~mm} \mathrm{Hg}$ ), and refraction (SD 1.5 dioptres spherical equivalent). The study has been approved by the appropriate institutional review board, and informed consent was obtained from each patient/volunteer following complete explanation of the procedure.

OPA is an indirect measure of choroidal perfusion. A bolus of blood entering the eye corresponding to the cardiac pulse represents a change in IOP as a function of time reflecting the pulsatile component (approximately $70 \%)^{21}$ of intraocular blood flow, ${ }^{22} 85 \%$ of which is choroidal. ${ }^{11}$

The technology of the ocular blood flow (OBF) system (OBF Labs Ltd, Wilts) and its relation to choroidal blood flow has been described in detail elsewhere. ${ }^{23}$ In addition to pneumotonometric values, IOP was measured by Goldmann applanation tonometry. Brachial artery pressures were measured non-invasively (Riva-Rocci) using a blood pressure cuff and a stethoscope. Heart rate (HR) was measured manually using a stopwatch. Applanation IOP, systolic (BPsyst) and diastolic (BPdiast) blood pressures (BP) and HR were taken before OPA measurements.

STATISTICS

All data represent arithmetic mean (SEM); Student's unpaired two tailed $t$ test and linear regression analysis were used for statistical analysis; correlation ( $r$ ) and regression (q) coefficients were calculated. Measurements were performed on both eyes of each subject. The level of significance was 5\%. RP groups were compared with matched subgroups from a pool of healthy volunteers and with each other.

\section{Results}

When compared with matched subgroups from a pool of healthy controls ( $\mathrm{n}=94$ ), neither IOP (Fig 1, Table 1) nor systemic perfusion parameters (Table 1) were significantly $(p>0.1)$ altered in any of the three RP groups investigated.

OPA (mm Hg; Fig 2, Tables 1 and 2) in RP-I (2.2 (0.1)) was within the same range as the controls $(2.2(0.2))$. However, in all other RP groups, OPA was highly significantly reduced. At stage II (1.6 (0.1)) the reduction was $27.3 \%$ $(\mathrm{p}<0.0001)$ further declining-corresponding to the severity of the disease-to a $45.5 \%$ reduction $(\mathrm{p}<0.0001)$ at stage RP-III $(1.2$ $(0.1))$. 
Table 2 Comparison of ocular pulse amplitudes (OPAs, mm Hg; mean (SEM)) of retinitis pigmentosa (RP) patients stages $I(n=22), I I(n=29)$, and III $(n=24)$, each compared with a matched control subgroup from a pool of healthy volunteers $(n=94)$ and with each other

\begin{tabular}{llll}
\hline & \multicolumn{3}{l}{ Statistics $(p$ values) } \\
\cline { 2 - 4 } & $R P-I$ & $R P-I I$ & $R P-I I I$ \\
\hline Control & 1.0 & $<0.0001$ & $<0.0001$ \\
RP-I & & 0.0002 & $<0.0001$ \\
RP-II & & & 0.0031 \\
\hline
\end{tabular}

${ }^{\star}$ Represents $\mathrm{p}<0.05$ using Student's unpaired two tailed $t$ test.

When compared with RP-I, OPA showed a $27.3 \%$ reduction $(p=0.0002)$ in RP-II and a $45.6 \%$ reduction $(\mathrm{p}<0.0001)$ in RP-III. RP-III showed a $25.0 \%(\mathrm{p}=0.0031)$ reduction when compared with RP-II.

Visual field size $\left(\mathrm{cm}^{2}\right)$ was $15.1(0.06), 11.01$ (0.43), $5.31(0.27)$, and $1.63(0.14)$ for healthy controls and RP-I to RP III, respectively.

Linear regression analysis showed a significant correlation for OPA and visual field size $(r$ $=0.99996, q=9.39, p=0.0028)$.

\section{Discussion}

The lack of evidence of pathological changes in the retinal pigmented epithelium (RPE) and choroidal vasculature in the initial stage of the disease as demonstrated by fluorescein angiography $^{24}$ and by the study presented here may indicate that the pathological process is initiated in the photoreceptors and that vascular changes may evolve secondary to photoreceptor degeneration.

Morphological findings in advanced RP show rods and cones clustered in irregularly shaped patches, in association with pigmented retinal epithelial cells in the macula with only cones in the middle of these patches showing poorly organised outer segments. Only few photoreceptors remain outside of the macula with the RPE being relatively normal near surviving photoreceptors, and depigmented, thinned, or missing in areas of photoreceptor loss. ${ }^{25}$ Indocyanine green angiography demonstrates hypofluorescence in the chorioretinal atrophic areas ${ }^{26}$ indicating reduced perfusion in retinal $^{2}$ and choroidal vascular systems as reflected by the reduction in OPA demonstrated in the present study and further supported by morphological ${ }^{5}$ and videoangiographic studies ${ }^{6}$ showing absence or atrophy of the choriocapillaris in RP.

Similar low OPAs were observed in patients with $\mathrm{NTG}^{13}{ }^{16}$ and diabetic retinopathy, ${ }^{27}$ both being ocular pathology where vascular capacity is lost due to vascular degeneration and/or vascular dysregulation.

Clearly, as OPA is reduced at first at stage $\mathrm{RP}-\mathrm{II}$ and as at least in two other types of ocular pathology as well, ${ }^{13} 1627$ it can be used neither for clinical diagnosis of RP nor to follow the natural course of the disease. However, the data show that in advanced stages of RP not only the RPE and retina but also the functional integrity of the choroid are affected by this genetic aberration, and OPA may be an indicator of degenerative changes in this vascular structure.
Since nitric oxide (NO), a potent vasodilator produced in the retina, ${ }^{28} \mathrm{RPE},{ }^{29}$ as well as ophthalmic and ciliary arteries, ${ }^{30}$ is involved in the regulation of choroidal vascular tone, ${ }^{31}$ a destruction of the RPE and retina may lead to an impeded production of this metabolite, resulting in vasoconstriction and even obliteration of choroidal and retinal vessels as a secondary phenomenon due to the RP disease process in the RPE and retina.

A consequence from our data may be to use vasodilators in RP in order to quantify their influence on ocular perfusion and visual field size and to correlate both parameters in a long term double blind randomised crossover placebo controlled study. Before starting in humans, the OPA alteration found in RP patients should be proved in an RP animal model-for example, in the RCS rat. This will allow us to follow the course of OPA in parallel to progression of the disease and to correlate OPA measurements with histological changes in the RPE, retina, and choroid. In a future series of experiments vasodilators should be investigated.

This may also help to clarify whether choroidal ischaemia contributes as a primary factor to progression of RP or is (as discussed above) caused secondly as a result of mediators released in the RPE and/or retina. In either case dilating choroidal vessels should increase OPA in advanced RP and thus-as demonstrated with a carbon dioxide provocation test $^{20}$ - possibly improve visual field size.

Care must be taken in choosing the vasodilator suited best for such a study since vasodilators in general do not necessarily increase ocular perfusion.

Nitrates, such as isosorbide dinitrate (ISDN), cause vasodilatation, especially in the veins. Nitrate induced peripheral venous pooling reducing cardiac preload is well investigated. ${ }^{32}$ This and the direct spasmolytic effect on coronary arteries ${ }^{33}$ are the basis for the wide use of nitrates in coronary artery disease. ${ }^{32} 33$ Frayser and Hickam ${ }^{34}$ noted arterial and venous dilatation in retinal vessels following application of nitrates. Surprisingly, oxygen saturation was reduced in the retinal venules, and the arteriovenous oxygen difference was increased, interpreted by the authors as a reduction in retinal perfusion. This finding might be explained by local vasoconstriction due to oxygen ${ }^{35}$ and/or the drop in systolic ophthalmic artery pressure, systolic ocular perfusion pressure and OPA, passively reflecting the drop in systemic blood pressure. ${ }^{36}$

Nifedipine and nimodipine are dihydropyridine based calcium channel blockers with different tissue selectivities for vascular smooth muscle cells. Nifedipine acts primarily on coronary and peripheral vessels and is used in coronary heart disease, systemic hypertension, and Raynaud's disease, whereas centrally acting nimodipine is primarily used in neurology and neurosurgery to treat acute and chronic ischaemic cerebrovascular damage, avoiding systemic hypotensive side effects.

Nifedipine is applied in patients with NTG, increasing reduced choroidal perfusion ${ }^{16}$ and 
improving visual field size in selected patients following acute and chronic dosing. ${ }^{19}$ These haemodynamic findings might explain the potential stabilising effect of nifedipine on visual fields in NTG patients. Nimodipine improves visual function in some HTG, NTG, and even in healthy volunteers, but surprisingly capillary optic nerve head blood flow as measured by laser Doppler flowmetry according to Riva was unchanged, ${ }^{37}$ suggesting an improvement in visual function after application of nimodipine at least in healthy volunteers independent of an increase in optic nerve head blood flow which may be related to an increase in retinal or (as with nifedipine) choroidal blood flow or to a reduction in neuronal intracellular calcium fluctuations. ${ }^{38}$ However, neither calcium channel blocker has been investigated in RP.

The contrasting effects of ISDN and nifedipine on ocular perfusion might be explained by different pharmacological modes of action. Nitrates act via pronounced vasodilatation of venous capacity vessels by liberating nitric oxide, which is also an endogenous vasodilator from the vascular endothelium, ${ }^{32}{ }^{39}$ whereas calcium channel blockers primarily reduce arterial resistance by blocking calcium influx into the vascular smooth muscle cell ${ }^{40}$ and by inhibiting the endothelial vasoconstrictor, endothelin- $1 .^{41}$

\section{Conclusion}

OPA, a haemodynamic parameter reflecting mainly choroidal perfusion, is reduced in RP patients beginning at stage II, which differs from another observation ${ }^{7}$ reporting on an overall reduction of OPA without correlating it to stage of disease.

Because OPA is also reduced in other pathology, ${ }^{13} 1627$ and reduction is not significant up to stage II, it can be used neither as a specific measure for clinical detection of RP nor to follow the natural course of this disease. However, the data show that in advanced stages of RP not only the RPE and retina but also the choroid are affected by this genetic aberration.

The apparent decrease of choroidal capacity in RP suggests a vasodilatory approach to the choroid, avoiding a "steal" effect.

Thus, a long term nitrate therapy cannot be enhanced by ophthalmocirculatory aspects. A negative effect on progression of retinal degeneration in RP patients who are also on nitrates for other disorders is conceivable. In contrast with nitrates, nifedipine and other primarily arterial vasodilators might increase ocular, mainly choroidal, perfusion and thus improve nourishment to and removal of potential toxic waste products from the RPE and the adjacent non-vascularised outer layers of the retina, macula, and optic disc regions in RP patients.

Future controlled studies on vasoactive drugs will elucidate the effect of increased ocular perfusion on progression of this disease.

1 Berson EL. Retinitis pigmentosa. Invest Ophthalmol Vis Sci 1993;34:1659-76.
2 Grunwald JE, Maguire AM, Dupont J. Retinal hemodynamics in retinitis pigmentosa. Am $\mathcal{f}$ Ophthalmol 1996;122:5028.

3 Wagner $U$, Wiederholt $M$. Membrane voltage and wholecell currents in cultured perizytes of control rats and rats with retinal dystrophy. Curr Eye Res 1996;15:1045-53

4 Flannery JG, Farber DB, Bird AC, et al. Degenerative changes in a retina affected with autosomal dominant retinitis pigmentosa. Invest Ophthalmol Vis Sci 1989;30: 191-211.

5 Henkind P, Gartner S. The relationship between retinal pigment epithelium and the choriocapillaris. Trans Ophthalmol Soc UK 1983;103:444-7.

6 Ulrich C, Ulrich WD, Vehlow K, et al. Haemodynamic aspects of retinitis pigmentosa. Fortschr Ophthalmol 1991; 88:642-7.

7 Langham ME, Kramer T. Decreased choroidal blood flow associated with retinitis pigmentosa. Eye 1990;4:374-81.

8 Alm A, Bill A. The oxygen supply to the retina. II. Effects of high intraocular pressure and increased arterial carbon dioxide tension on uveal and retinal blood flow in cats. Acta dioxide tension on uveal and reting
Physiol Scand 1972;84:306-19.

9 Alm A, Bill A. Ocular and optic nerve head blood flow at normal and increased intraocular pressures in monkeys (Macaca iris): a study with radioactively labelled microspheres including flow determinations in brain and some other tissues. Exp Eye Res 1973;15:15-29.

10 Törnquist P, Alm A. Retinal and choroidal contribution to retinal metabolism in vivo. A study in pigs. Acta Physiol Scand 1979;106:351-7.

$11 \mathrm{Alm}$ A. Ocular circulation. In: Hart WM, ed. Adler's physiology of the eye. St Louis: CV Mosby, 1992:198-227.

12 Bill A. Blood circulation and fluid dynamics in the eye. Physiol Rev 1975;55:383.

13 Schmidt KG, v Rückmann A, Mittag TW, et al. Reduced ocular pulse amplitude in low tension glaucoma is ocular pulse amplitude in low tension gla

14 Schmidt KG, v Rückmann A, Mittag TW. Okuläre Pulsamplitude bei okulärer Hypertension und verschiedenen Glaukomformen. Ophthalmologica 1998;212:5-10.

15 Schmidt KG, Mittag TW, Pavlovic S, et al. Influence of physical exercise and nifedipine on ocular pulse amplitude. Graefes Arch Clin Exp Ophthalmol 1996;234:527-32.

16 Schmidt KG, v Rückmann A, Geyer O, et al. Einfluß des Nifedipins auf die okuläre Pulsamplitude bei Normaldruckglaukom. Klin Monatsbl Augenheilkd 1997;210: 355-9.

17 Schmidt KG, v Rückmann A, Pillunat LE. Dorzolamide increases ocular pulse amplitude in high tension primary open angle glaucoma. Br f Ophthalmol 1998;82:758-62.

18 Schmidt KG. Antiglaukomatosa und choroidale Perfusion bei primärem und experimentell induziertem Offenwinkelglaukom. In: Schmidt KG, Pillunat LE, eds. Fortbildung Glaukom, Bd 1, Perfusion und Pharmakologie. Stuttgart: Enke, 1999:39-50.

19 Gasser P, Flammer J. Short- and long-term effect of nifedipine on the visual field in patients with presumed vasospasm. F Int Med Res 1990;18:334-9.

20 Tacke CM, Pillunat LE, Lang GK. Okulare Kohlendioxidreaktivität bei Retinopathia pigmentosa. Perimetrische Ergebnisse. Ophthalmologe 1993;90:510-4.

21 Williamson TH, Harris A. Ocular blood flow measurement. Br f Ophthalmol 1994;78:939-45.

22 Silver DM, Farrell RA. Validity of pulsatile ocular blood flow measurements. Surv Ophthalmol 1994;38(Suppl):72-80.

23 Schmidt KG. Basic principle of the OBF-System. In: Pillunat LE, Harris A, Anderson DR, Greve EL, eds. Current concepts on ocular blood flow in glaucoma. The Hague: concepts on ocular blo
Kugler, 1999:75-95.

24 Grondahl J. Pericentral retinal dystrophy. Acta Ophthalmol Copenh 1987;65:344-51.

25 Tucker GS, Jacobson SG. Morphological findings in retinitis pigmentosa with early diffuse rod dysfunction. Retina 1988;8:30-41

26 Nagai Y, Matsubara T, Takahashi K, et al. Angiographic findings and histological localization of indocyanine green in N-methyl-N-nitrosourea induced retinal degeneration in rats. Nippon Ganka Gakkai Zasshi 1999;103:489-96.

27 Langham ME, Grebe R, Hopkins M, et al. Choroidal blood flow in diabetic retinopathy. Exp Eye Res 1991;52:167-73.

28 Osborne NN, Barnett NL, Herrera AJ. NADPH diaphorase localisation of nitric oxide synthetase activity in retina and anterior uvea. Brain Res 1993;610:194-8.

29 Gourneau O, Hicks D, Courtouis Y. Human retinal pigmented epithelial cells produce nitric oxide in response to cytocines. Biochem Biophys Res Comm 1994;198:120-6.

30 Haefliger IO, Flammer J, Luscher TF. Heterogeneity of endothelium-dependent regulation in ophthalmic and ciliary arteries. Invest Ophthalmol Vis Sci 1993;34:1722-30.

31 Schmidt KG, Mittag TW, Geyer O, et al. Choroidal vascular tone: potential biochemical-pharmacological mechanisms. Exp Eye Res (in press)

32 Anderson TJ, Meredith IT, Ganz P, et al. Nitric oxide and nitrovasodilators: similarities, differences, and potential interactions. F Am Coll Cardiol 1994;24:555-66.

33 Metha JL. Endothelium, coronary vasodilation, and organic nitrates. Am Heart f 1995;129:382-91.

34 Frayser R, Hickam JB. Effect of vasodilator drugs on the retinal blood flow in man. Arch Ophthalmol 1965;73:640-2.

35 Frayser R, Saltzman HA, Anderson B Jr, et al. The effect of hyperbaric oxygenation on retinal circulation. Arch Ophthalmol 1967;77:265-9.

36 Hessemer V, Schmidt KG. Influence of isosorbide dinitrate on ocular circulation. Arch Ophthalmol 1997;115:324-7. 
37 Christ A, Pillunat LE, Kuba GB, et al. Contrast sensitivity and optic nerve head blood flow after application of calcium-channel blockers (abstract). Invest Ophthalmol Vis Sci 1996;37:1251

38 Abele AE, Scholz KP, Scholz WK, et al. Excitotoxicity induced by enhanced excitatory neurotransmission in cultured hippocampal pyramidal neurons. Neuron 1990;4 413-9.

39 Abe S, Nishimura J, Nakamura M, et al. Effects of nicorandil on cytosolic calcium concentrations and on tension development in the rabbit femoral artery. 7 Pharmacol Exp Ther 1994;268:762-71.

40 Shrier I, Magder S. Effects of nifedipine on vascular waterfall and arterial resistance in canine hindlimb. Am F Physiol 1995;268:371-6.

41 Nyborg NCB, Prieto D, Benedito S, et al. Endothelin-1 induced contraction of bovine retinal small arteries is reversible and abolished by nitrendipine. Invest Ophthalmol Vis Sci 1991;32:27-31.

\section{Video Reports (www.bjophthalmol.com)}

Capsule staining and mature cataracts: a comparison of indocyanine green and trypan blue dyes. D F Chang

Pearls for implanting the Staar toric IOL. D F Chang

An intraocular steroid delivery system for cataract surgery. D F Chang 